\title{
PAIR EXCITATIONS AND VERTEX CORRECTIONS IN FERMI FLUIDS AND THE DYNAMIC STRUCTURE FUNCTION OF TWO-DIMENSIONAL ${ }^{3} \mathrm{He}$
}

\author{
H. M. Böhm* $\dagger$, H. Godfrin ${ }^{\ddagger}$, E. Krotscheck $\dagger$, H. J. Lauter ${ }^{\#}$, M. Meschke ${ }^{\ddagger}$, and M. Panholzer ${ }^{\dagger}$ \\ Inst. f. Theoretische Physik, Johannes Kepler Universität, \\ A-4040 Linz, Austria \\ *E-mail: helga.boehm@jku.at \\ $¥$ Centre de Recherches sur les Tres Basses Temperatures, \\ C.N.R.S., B.P. 166X, 38042 Grenoble, France \\ \# Institute Laue Langevin, 38042 Grenoble Cedex, France.
}

\begin{abstract}
We use the equations-of-motion approach for time-dependent pair correlations in strongly interacting Fermi liquids to develop a theory of the excitation spectrum and the single-particle self energy in such systems. We present here the fully correlated equations and their approximate solutions for ${ }^{3} \mathrm{He}$. Our theory has the following properties: It reduces to both, i) the "correlated" random-phase approximation (RPA) for strongly interacting fermions if the two-particle-two-hole correlations are ignored, and, ii) to the correlated Brillouin-Wigner perturbation theory for boson quantum fluids in the appropriate limit. iii) It preserves the two first energy-weighted sum rules, and systematically improves upon higher ones. iv) A familiar problem of the standard RPA is that it predicts a roton energy that lies more than a factor of two higher than what is found in experiments. A popular cure for this is to introduce an effective mass in the Lindhard function. No such $a d-h o c$ assumption is invoked in our work. We demonstrate that the inclusion of correlated pair-excitations improves the dispersion relation significantly. Finally, a novel form of the density response function is derived that arises from vertex corrections in the proper polarization.
\end{abstract}

Keywords: Fermi fluids, density response function, multi-pair excitations, ${ }^{3} \mathrm{He}$, correlated basis functions

\section{Introduction}

The method of choice for studying the strongly interacting quantum liquids ${ }^{3} \mathrm{He}$ and ${ }^{4} \mathrm{He}$ is the Jastrow-Feenberg variational method and its extension in the form of correlated basis functions (CBF) theory. ${ }^{1}$ The theory builds the correlations of the system into the the basis of the Hilbert space in a natural and straightforward manner. While ground state properties are accessible in many cases via simulation studies, the above approach remains the most powerful tool for a quantitatively reliable prediction of dynamic properties. In the last decade the excitation spectrum of the Bose system has been successfully investigated for a variety of configurations, 
ranging form the basic works of Refs. 2,3 to recent studies of the influence of phonon decay on the transport current. ${ }^{4}$ In all these cases the inclusion of dynamic, i.e. time-dependent pair correlations has proved necessary. It is not only plausible, but has been demonstrated convincingly in several cases, that short-ranged fluctuations become relevant for excitations with wavelengths that are comparable to the interparticle distance. A special case of such pair excitations was already introduced by Feynman ${ }^{5}$ in the form of "backflow" effects.

The theory of pair fluctuations is very well understood for Bose systems, but is still in its beginnings for fermions. We expect that also in the the case of Fermi systems, especially in ${ }^{3} \mathrm{He}$ whose properties are dominated by the strong, shortranged repulsion, the dispersion of phonons is visibly influenced by short-ranged fluctuations. In addition, accounting for these processes is indispensable for a description of the damping of long-wavelength phonons. This work reports progress in our development of a theory for bulk ${ }^{3} \mathrm{He}$ with the ultimate goal of reaching a level of accuracy as well as consistency comparable to the theory for bosons.

Central to our investigations is the density-density response function $\chi(q, \omega)$ connected to the dynamic structure function by

$$
S(q, \omega)=-\frac{1}{\pi} \Im m \chi(q, \omega) .
$$

In Fermi liquids $\chi(q, \omega)$ is often taken to be of the "RPA" form

$$
\chi^{\mathrm{RPA}}(q, \omega)=\frac{\chi_{0}(q, \omega)}{1-\widetilde{V}_{\mathrm{p}-\mathrm{h}}(q) \chi_{0}(q, \omega)},
$$

where $\chi_{0}$ is the Lindhard function and $\widetilde{V}_{\mathrm{p}-\mathrm{h}}(q)$ a suitable effective interaction (also termed, "pseudo-potential ${ }^{6 "}$ and "local field corrected potential" "). One can determine $\widetilde{V}_{\mathrm{p}-\mathrm{h}}(q)$ through the $\omega^{0}$ and $\omega^{1}$ sum rules, but this leads to a collective mode that is energetically much higher than what is found in experiments. An ad-hoc repair of this problem is to introduce an effective mass ${ }^{8} m^{*}$ in $\chi_{0}$. The effective mass in both three- and two-dimensional ${ }^{3} \mathrm{He}$ is far from constant, ${ }^{9,10}$ it exhibits a strong peak around the Fermi momentum $k_{F}$, and another one at approximately $2.5 k_{F}$. Introducing an $m^{*}$ is unsatisfactory mainly from two points of view: First, we want to develop a manifestly microscopic description of the low-temperature properties of ${ }^{3} \mathrm{He}$, and second, it introduces inconsistencies because the important sum rules are violated, which makes $\widetilde{V}_{\mathrm{p}-\mathrm{h}}(q)$ undetermined. The above example of a successful description of the phonon-roton spectrum in ${ }^{4} \mathrm{He}$ systems - which does not even introduce the concept of an "effective mass" - leads one to expect that the same physical mechanisms, viz. dynamic pair correlations, lower the phonon-roton spectrum not only in ${ }^{4} \mathrm{He}$ but also in ${ }^{3} \mathrm{He}$, without violating the two above sum rules.

An even more compelling argument against introducing an effective mass is provided by experimental data on the dynamic structure function of two-dimensional ${ }^{3} \mathrm{He}$. An example of the experimental data is shown in Fig. 1. Here the collective 
mode is found inside the particle-hole continuum. ${ }^{11}$ It would be quite difficult to explain the feature that the maximum strength of a possible collective mode is found inside the continuum with the form (2) of the density density response function, in particular this feature cannot be reproduced by changing $m^{*}$ in the RPA formula $(2)$.

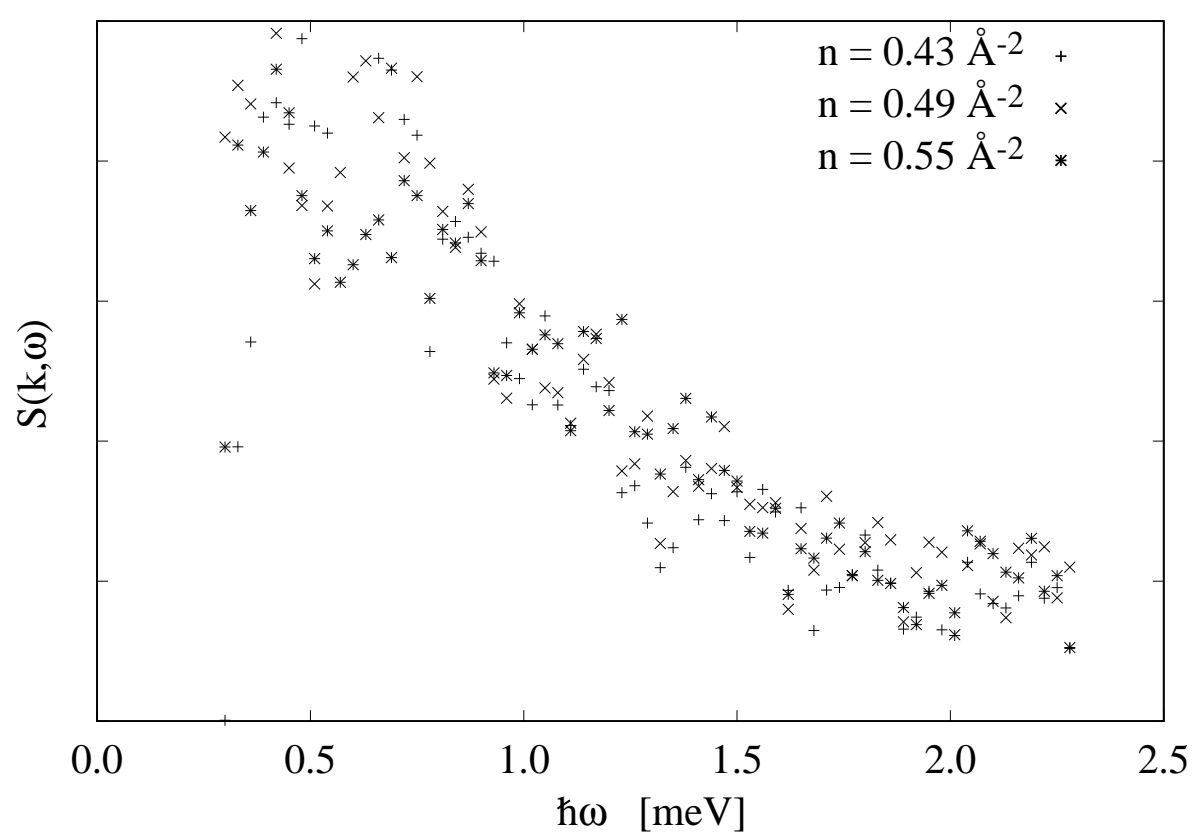

Fig. 1. The figure shows experimental data of the dynamic structure function $S(k, \omega)$ in twodimensional ${ }^{3} \mathrm{He}$ for wave numbers $1.1 \AA^{-1} \leq k \leq 1.2 \AA^{-1}$. These data were obtained by three of us (H. G., H.-J. L and M. M.) at the IN14 instrument at the Institute Laue-Langevin in Grenoble.

\section{Equations of motion}

\subsection{Correlated equations of motion}

We describe the excited state wave function of strongly interacting fermions by a generalized time-dependent Hartree-Fock form

$$
|\Psi(t)\rangle=\frac{1}{\sqrt{\mathcal{N}}} e^{-i t H_{00} / \hbar} F e^{U(t)}\left|\Phi_{0}\right\rangle
$$

where $\mathcal{N}$ denotes the normalization integral, $\left|\Phi_{0}\right\rangle$ the ground state of a model system (normally a Slater determinant) and $H_{00}$ the energy of the correlated ground state $F\left|\Phi_{0}\right\rangle$. The most general excitation operator $U$ contains $n$-particle amplitudes 
$u_{(n)}(t)$

$$
\begin{aligned}
U & =\sum_{n} \sum_{\substack{\mathbf{p}_{\mathbf{p}_{1} \ldots \mathbf{p}_{n}} \\
\mathbf{h}_{1} \ldots \mathbf{h}_{n}}} u_{\mathbf{p}_{1} \ldots \mathbf{p}_{n} \mathbf{h}_{1} \ldots \mathbf{h}_{n}}(t) a_{\mathbf{p}_{1}}^{\dagger} \ldots a_{\mathbf{p}_{n}}^{\dagger} a_{\mathbf{h}_{n}} \ldots a_{\mathbf{h}_{1}} \\
& \equiv \sum_{n} \sum_{(p h)^{n}} u_{(n)}(t) \widehat{X}_{n} .
\end{aligned}
$$

These amplitudes are determined by minimizing the action integral $\mathcal{S}=\int d t \mathcal{L}$ corresponding to the time-dependent Schrödinger equation:

$$
\left[\frac{\delta}{\delta u_{(n)}^{*}}-\frac{d}{d t} \frac{\delta}{\delta \dot{u}_{(n)}^{*}}\right] \mathcal{L}=0 \quad \text { with } \quad \mathcal{L}=\left\langle\Psi\left|H+H^{\operatorname{ext}}(t)+\frac{\hbar}{i} \frac{\partial}{\partial t}\right| \Psi\right\rangle .
$$

Here, $H^{\text {ext }}(t) \equiv \int d^{3} r h^{\text {ext }}(\mathbf{r} ; t) \hat{\rho}(\mathbf{r})$ describes a small and real external perturbation where $\hat{\rho}(\mathbf{r})$ is the density operator. Considering $H^{\text {ext }}$ as a perturbation, linear equations of motion (EOMs) are derived by expanding $\mathcal{L}$ to order $\left(U^{\dagger}\right)^{2}$. Using the abbreviation $H^{\prime} \equiv H-H_{00}$ and denoting expectation values taken in the free ground state $\left\langle\Phi_{0}|\ldots| \Phi_{0}\right\rangle$ by $\langle\ldots\rangle_{0}$ these EOMs read

$$
\begin{gathered}
-\frac{\hbar}{i}\left(\left\langle\widehat{X}^{\dagger} F^{\dagger} F \dot{U}\right\rangle_{0}-\left\langle\widehat{X}^{\dagger} F^{\dagger} F\right\rangle_{0}\left\langle F^{\dagger} F \dot{U}\right\rangle_{0}\right)=\left\langle\widehat{X}^{\dagger} F^{\dagger} H^{\mathrm{ext}} F\right\rangle_{0}-\left\langle F^{\dagger} H^{\mathrm{ext}} F\right\rangle_{0} \\
+\left\langle\widehat{X}^{\dagger} F^{\dagger} H^{\prime} F U\right\rangle_{0}+\left\langle\widehat{X}^{\dagger} U^{\dagger} F^{\dagger} H^{\prime} F\right\rangle_{0}-\left\langle U^{\dagger} F^{\dagger} H^{\prime} F\right\rangle_{0}\left\langle\widehat{X}^{\dagger} F^{\dagger} F\right\rangle_{0} .
\end{gathered}
$$

In the standard notation of correlated basis functions (CBF) formalism ${ }^{12}$

$$
\begin{aligned}
& \frac{\left\langle\widehat{X}_{n}^{\dagger} F^{\dagger} H^{\prime} F \widehat{X}_{n^{\prime}}^{\prime}\right\rangle_{0}}{\left\langle F^{\dagger} F\right\rangle_{0}}=H_{X_{n}, X_{n^{\prime}}^{\prime}}^{\prime} z_{X_{n}} z_{X_{n^{\prime}}^{\prime}}:= \\
& \frac{\left\langle F \widehat{X}_{n} \Phi_{0}\left|H^{\prime}\right| F \widehat{X}_{n^{\prime}}^{\prime} \Phi_{0}\right\rangle}{\sqrt{\left\langle\widehat{X}_{n}^{\dagger} F^{\dagger} F \widehat{X}_{n}\right\rangle_{0}\left\langle\widehat{X}_{n^{\prime}}^{\dagger \dagger} F^{\dagger} F \widehat{X}_{n^{\prime}}^{\prime}\right\rangle_{0}}} \frac{\sqrt{\left\langle\widehat{X}_{n}^{\dagger} F^{\dagger} F \widehat{X}_{n}\right\rangle_{0}}}{\sqrt{\left\langle F^{\dagger} F\right\rangle_{0}}} \frac{\sqrt{\left\langle\widehat{X}_{n^{\prime}}^{\prime \dagger} F^{\dagger} F \widehat{X}_{n^{\prime}}^{\prime}\right\rangle_{0}}}{\sqrt{\left\langle F^{\dagger} F\right\rangle_{0}}}
\end{aligned}
$$

and the analogous convention for the off-diagonal normalization integrals

$$
\frac{\left\langle\widehat{X}_{n}^{\dagger} F^{\dagger} F \widehat{X}_{n^{\prime}}^{\prime}\right\rangle_{0}}{\left\langle F^{\dagger} F\right\rangle_{0}} \equiv N_{X_{n}, X_{n^{\prime}}^{\prime}} z_{X_{n}} z_{X_{n^{\prime}}^{\prime}}
$$

the EOMs take the form

$$
\begin{aligned}
& \sum_{n^{\prime}} \sum_{\left(p^{\prime} h^{\prime}\right)^{n^{\prime}}}\left\{-\frac{\hbar}{i} \dot{u}_{\left(n^{\prime}\right)}\left(z_{X_{n^{\prime}}^{\prime}} N_{X_{n}, X_{n^{\prime}}^{\prime}}-z_{X_{n^{\prime}}^{\prime}} N_{X_{n}, 0} N_{0, X_{n^{\prime}}^{\prime}}\right)\right\}=H_{X_{n}, 0}^{\text {ext }}+ \\
& \sum_{n^{\prime}} \sum_{\left(p^{\prime} h^{\prime}\right)^{n^{\prime}}}\left\{u_{\left(n^{\prime}\right)} z_{X_{n^{\prime}}^{\prime}} H_{X_{n}, X_{n^{\prime}}^{\prime}}^{\prime}+u_{\left(n^{\prime}\right)}^{*}\left(z_{X_{n^{\prime}}^{\prime}} H_{X_{n} X_{n^{\prime}}^{\prime}, 0}^{\prime}-z_{X_{n^{\prime}}^{\prime}} N_{X_{n}, 0} H_{X_{n^{\prime}}^{\prime}, 0}^{\prime}\right)\right\}
\end{aligned}
$$

(for a uniform system, we have $z_{X_{n} X_{n^{\prime}}^{\prime}}=z_{X_{n}} z_{X_{n^{\prime}}^{\prime}}+\mathcal{O}\left(N^{-1}\right)$ ). Technically, we restrict ourselves to single particle and pair fluctuations. We spell out these equations in detail for single-particle and pair fluctuations in the appendix; here we adopt a more pragmatic strategy: In essence, the CBF theory provides a means ${ }^{13,14}$ to map 
the strong, bare interaction of the system onto weak, effective interactions. This mapping is uniquely determined by the diagrammatic analysis of off-diagonal correlated matrix elements, ${ }^{15}$ generally one can define non-local effective interactions from overlap integrals of the Hamiltonian between correlated states (as examples we mention $H_{p h, p^{\prime} h^{\prime}}$ and $H_{h h^{\prime} p p^{\prime}, h h^{\prime} p^{\prime \prime} p^{\prime \prime}}$; which, in turn, defines a pair operator $\mathcal{H}$ via the anti-symmetrized expectation values $\left\langle p h^{\prime}|\mathcal{H}| h p^{\prime}\right\rangle_{\mathrm{a}}$, and $\left.\left\langle p p^{\prime}|\mathcal{H}| p^{\prime \prime} p^{\prime \prime \prime}\right\rangle_{\mathrm{a}}\right)$. Once this mapping is performed, the originally strongly interacting system can be regarded as only moderately interacting in these new effective interactions.

\subsection{The Hartree Fock (HF) limit}

To see the content of our theory of excitations at a somewhat simpler level, we treat the problem in this section in the Hartree-Fock approximation, i.e. the correlation operator $F$ is replaced by unity: $F, F^{\dagger} \rightarrow 1$. Consequently, all $z$-factors become 1 and the normalization overlap integrals are antisymmetrized delta-functions.

In the spirit of the ideas outlined at the end of Sec. 2.1 the interaction in this HF approximation should clearly be interpreted as an effective interaction which will ultimately be obtained from the correlated theory. We also should allow for different effective interactions in different channels. Denoting empty (occupied) states of the Slater determinant by $p_{i}=$ "particles" ( $h_{i}=$ "holes"), the "particle-hole interaction" $\widetilde{V}_{\mathrm{p}-\mathrm{h}}$ arises from matrix elements of the form $\left\langle h_{1} p_{2}|\mathcal{H}| p_{1} h_{2}\right\rangle$, whereas we would have a "particle-particle" interaction $\widetilde{V}_{\mathrm{p}-\mathrm{p}}(r)$ arising from matrix elements of the type $\left\langle p_{1} p_{2}|\mathcal{H}| p_{1}^{\prime} p_{2}^{\prime}\right\rangle$ (and analogously for "hole-hole" terms). Finally, another effective interaction $W$ arises from all other (=mixed) channels. Anticipating such effective interactions (all of which are normally non-local), we start with inspecting the EOMs for a weakly interacting system.

Eqs. (9) simplify to

$$
\frac{-\hbar}{i} \mathcal{A} \dot{u}_{(n)}=H_{X_{n}, 0}^{\mathrm{ext}}+\sum_{n^{\prime}} \sum_{\left(p^{\prime} h^{\prime}\right)^{n^{\prime}}}\left\{u_{\left(n^{\prime}\right)} H_{X_{n}, X_{n^{\prime}}^{\prime}}^{\prime}+u_{\left(n^{\prime}\right)}^{*} H_{X_{n} X_{n^{\prime}}^{\prime}, 0}^{\prime}\right\},
$$

where $\mathcal{A}$ denotes antisymmetrisation in all indices. In our case the fluctuation operator $U(t)$ in Eq. (4) consists of $1 \mathrm{p}-1 \mathrm{~h}$ and $2 \mathrm{p}-2 \mathrm{~h}$ fluctuations

$$
\begin{array}{ll}
\widehat{X}_{1}=a_{\mathbf{p}}^{\dagger} a_{\mathbf{h}} & u_{(1)}(t)=: c_{p h} \\
\widehat{X}_{2}=a_{\mathbf{p}}^{\dagger} a_{\mathbf{p}^{\prime}}^{\dagger} a_{\mathbf{h}^{\prime}} a_{\mathbf{h}} & u_{(2)}(t)=: \frac{1}{2} d_{p p^{\prime}, h h^{\prime}} .
\end{array}
$$

The pair correlations are symmetric under a pairwise interchange of indices, antisymmetry needs to be accounted for the hole (or particle) indices alone:

$$
d_{p p^{\prime}, h h^{\prime}}=d_{p^{\prime} p, h^{\prime} h} ; \quad \quad d_{p p^{\prime},\left(h h^{\prime}\right)_{a}} \equiv d_{p p^{\prime}, h h^{\prime}}-d_{p p^{\prime}, h^{\prime} h}
$$

Abbreviating the particle-hole excitation energies with $e_{p h} \equiv e_{p}-e_{h}$ the EOMs read 
explicitly

$$
\begin{aligned}
& {\left[i \hbar \frac{\partial}{\partial t}-e_{p h}\right] c_{p h}(t)=h_{p h}^{\mathrm{ext}}(t)+\left\langle p h^{\prime}\left|\widetilde{V}_{\mathrm{p}-\mathrm{h}}\right| h p^{\prime}\right\rangle_{a} c_{p^{\prime} h^{\prime}}+} \\
& \left\langle p p^{\prime}\left|\widetilde{V}_{\mathrm{p}-\mathrm{h}}\right| h h^{\prime}\right\rangle_{a} c_{p^{\prime} h^{\prime}}^{*}+\left\langle p h^{\prime}|W| p^{\prime \prime} p^{\prime}\right\rangle_{a} d_{p^{\prime \prime} p^{\prime}, h h^{\prime}}-\left\langle h^{\prime \prime} h^{\prime}|W| h p^{\prime}\right\rangle_{a} d_{p p^{\prime}, h^{\prime \prime} h^{\prime}},
\end{aligned}
$$

and

$$
\begin{aligned}
& {\left[i \hbar \frac{\partial}{\partial t}-e_{p h}-e_{p^{\prime} h^{\prime}}\right] d_{p p^{\prime}, h h^{\prime}}=} \\
& \left\langle p p^{\prime}|W| h p^{\prime \prime}\right\rangle c_{p^{\prime \prime} h^{\prime}}+\left\langle p p^{\prime}|W| p^{\prime \prime} h^{\prime}\right\rangle c_{p^{\prime \prime} h}-\left\langle p h^{\prime \prime}|W| h h^{\prime}\right\rangle c_{p^{\prime} h^{\prime \prime}}-\left\langle h^{\prime \prime} p^{\prime}|W| h h^{\prime}\right\rangle c_{p h^{\prime \prime}} \\
& \quad+\left[\left\langle h^{\prime \prime} p^{\prime}\left|\widetilde{V}_{\mathrm{p}-\mathrm{h}}\right| p^{\prime \prime} h^{\prime}\right\rangle_{a} d_{p p^{\prime \prime}, h h^{\prime \prime}}-\left\langle h^{\prime \prime} p^{\prime}\left|\widetilde{V}_{\mathrm{p}-\mathrm{h}}\right| h p^{\prime \prime}\right\rangle_{a} d_{p p^{\prime \prime}, h^{\prime \prime} h^{\prime}}+\left(\begin{array}{c}
p \leftrightarrow p^{\prime} \\
h \leftrightarrow h^{\prime}
\end{array}\right)\right] \\
& \quad+\left\langle p p^{\prime}\left|\widetilde{V}_{\mathrm{p}-\mathrm{p}}\right| p^{\prime \prime} p^{\prime \prime \prime}\right\rangle d_{p^{\prime \prime} p^{\prime \prime \prime}, h h^{\prime}}+\left\langle h^{\prime \prime} h^{\prime \prime \prime}\left|\widetilde{V}_{\mathrm{h}-\mathrm{h}}\right| h h^{\prime}\right\rangle d_{p p^{\prime}, h^{\prime \prime} h^{\prime \prime \prime}}
\end{aligned}
$$

\section{Solution strategy}

Without loss of generality we assume harmonic time dependence for the perturbation

$$
h_{\mathrm{ext}}(\mathbf{r}, t)=h_{\mathrm{ext}}(\mathbf{r}, \omega)\left(e^{i \omega t}+e^{-i \omega t}\right)
$$

and consequently a similar form for the excitation amplitudes.

In this first implementation of the theory we aim towards a form that is, as far as possible, analytic and preserves the analytic structure and the physical content of $\mathrm{CBF}$ theory for Bose systems. We therefore neglect exchange effects and also contributions of the "ladder" type, and we restrict the set of two-pair amplitudes to functions that are matrix elements of a local operator

$$
\sum_{p p^{\prime} h h^{\prime}} d_{p h, p^{\prime} h^{\prime}} a_{p}^{\dagger} a_{p^{\prime}}^{\dagger} a_{h^{\prime}} a_{h} \longrightarrow \sum_{q q^{\prime}} d\left(\mathbf{q}, \mathbf{q}^{\prime}\right)\left[\hat{\rho}_{\mathbf{q}} \hat{\rho}_{\mathbf{q}^{\prime}}-\hat{\rho}_{\mathbf{q}+\mathbf{q}^{\prime}}\right]
$$

where $\hat{\rho}_{\mathbf{q}}$ is the density operator in momentum space. Comparing our theory to descriptions using Feynman diagrams, our approach corresponds to accounting for the proper polarization graphs shown in Fig. 2. Clearly, the graphs no. 3 and 5-7 represent vertex corrections, which are beyond the RPA (i.e. these terms are not properly accounted for in response functions of the form of Eq. (2)).

We further simplify the $c$ - $d$ coupling by replacing the amplitudes $c_{p h}(t)$ in Eq. (14) by their Fermi-sea averages. With these approximations the EOMs can be solved for $c_{p h}$ and $d_{p h, p^{\prime} h^{\prime}}$ which, in turn, yield the linear response wave function and thus the induced density $\delta \rho(\mathbf{r}, t)$.

Introducing

$$
\chi_{0}^{( \pm)}(q, \omega)= \pm \frac{1}{N} \sum_{h} \frac{n_{h} \bar{n}_{\mathbf{h}+\mathbf{q}}}{\hbar \omega \mp e_{h+q, h}+i 0^{+}}
$$




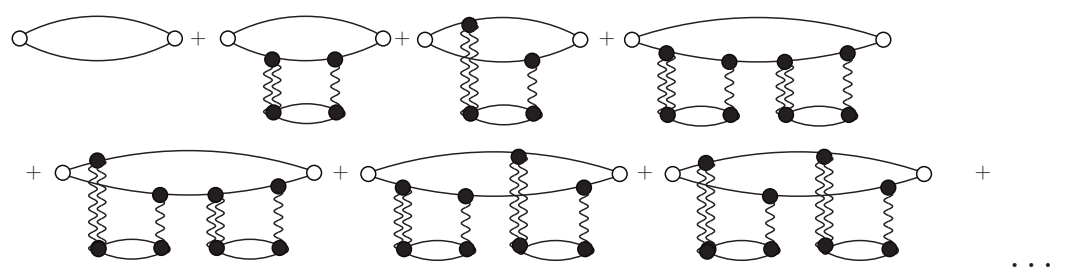

Fig. 2. Typical proper polarization Feynman diagrams taken into account in our approach. (The single and double wavy lines denote the bare and screened interactions, respectively. The lines denote free single particle propagators).

(where $n_{k}=\theta\left(k_{\mathrm{F}}-k\right) \equiv 1-\bar{n}_{k}$ ), the final result for the density-density response function can be expressed as follows:

$$
\begin{gathered}
\chi^{\text {Pair }}(q, \omega)=\frac{\kappa(q, \omega)}{1-\kappa(q, \omega) \widetilde{V}_{\mathrm{p}-\mathrm{h}}(q)} \\
\kappa(q, \omega)=\frac{\chi_{0}^{(+)}}{1-\chi_{0}^{(+)} \mathcal{W}_{+}}+\frac{\chi_{0}^{(-)}}{1-\chi_{0}^{(-)} \mathcal{W}_{-}}, \\
\mathcal{W}_{ \pm}=\mathcal{W}(q, \mp \omega)=-\frac{1}{2} \sum_{\mathbf{q}^{\prime}} \frac{\overline{\mathcal{W}}_{3}\left(\mathbf{q}-\mathbf{q}^{\prime}, \mathbf{q}^{\prime}\right) \overline{\mathcal{W}}_{3}\left(\mathbf{q}-\mathbf{q}^{\prime}, \mathbf{q}^{\prime}\right)}{ \pm \hbar \omega-\varepsilon\left(\mathbf{q}-\mathbf{q}^{\prime}\right)-\varepsilon\left(q^{\prime}\right)} .
\end{gathered}
$$

Here $\overline{\mathcal{W}}_{3}$ is a three- "phonon" vertex

$$
\overline{\mathcal{W}}_{3}\left(\mathbf{q}, \mathbf{q}^{\prime}\right)=\frac{\widetilde{V}_{\mathrm{p}-\mathrm{h}}(q) S_{F}(q)+\widetilde{V}_{\mathrm{p}-\mathrm{h}}\left(q^{\prime}\right) S_{F}\left(q^{\prime}\right)}{\sqrt{S_{\mathrm{F}}(q) S_{\mathrm{F}}\left(q^{\prime}\right)}} \frac{S_{\mathrm{F}}^{(3)}\left(\mathbf{q}, \mathbf{q}^{\prime}, \mathbf{q}+\mathbf{q}^{\prime}\right)}{S_{\mathrm{F}}\left(\mathbf{q}+\mathbf{q}^{\prime}\right)} .
$$

with $S_{\mathrm{F}}$ nd $S_{\mathrm{F}}^{(3)}$ denoting the static structure factors of the non-interacting Fermi gas. In $\varepsilon(q)$ we recover the weakly interacting limit of the Bogoljubov dispersion relation

$$
\begin{aligned}
\varepsilon(q) & =\frac{\hbar^{2} q^{2}}{2 m S_{\mathrm{F}}(q)} 1+S_{\mathrm{F}}(q) \widetilde{V}_{\mathrm{p}-\mathrm{h}}(q) \\
& \approx \frac{\hbar^{2} q^{2}}{2 m S_{\mathrm{F}}(q)} \sqrt{1+4 m S_{\mathrm{F}}^{2}(q) \widetilde{V}_{\mathrm{p}-\mathrm{h}}(q) / \hbar^{2} q^{2}} \approx \frac{\hbar^{2} q^{2}}{2 m S(q)}
\end{aligned}
$$

This dispersion relation (23) can be obtained, for example, by a "collective approximation" of the RPA equation (2).

There are two important differences between the density response function from the EOMs for the strongly interacting case and its weakly interacting counterpart. First, $\varepsilon(q)$ in Eq.(20) becomes the full Bogoljubov spectrum corresponding to the exact interacting structure factor $S(q)$, and, second, a multi-phonon contribution $\widetilde{V}_{\mathrm{mp}}(q, \omega)$ must be added in the denominator of Eq. (18). This latter term is of no immediately transparent structure and numerical investigations show that its inclusion has only a marginal effect on the numerical results. ${ }^{16}$ 
We end this section by noting that it is easily proved that $\chi^{\text {Pair }}(q, \omega)$ obeys the $\omega^{0}$ and $\omega^{1}$ sum rules, independently of the specifics of $\mathcal{W}(q, \omega)$. This means that $\widetilde{V}_{\mathrm{p}-\mathrm{h}}(q)$ is uniquely defined by the static structure function through the first two energy weighted sumrules.

\section{Applications to two-dimensional ${ }^{3} \mathrm{He}$}

We show in Fig. 3 results for the dynamic structure factor $S(k, \omega)$ of twodimensional ${ }^{3}$ He for an areal density of $n=0.049 \AA^{-2}$ and 4 different wave numbers in the vicinity of the experimental data. The effective interaction was calculated through the $\omega^{0}$ sum rule from the static $S(k)$ obtained by diffusion Monte Carlo calculations in Ref. 17. In the spirit of the above discussion, the multi-phonon contribution has been neglected for the time being.

Results are shown for the RPA as well as for the CBF theory in Fig. 3. The three figures for $k=0.85 \AA^{-1}, k=1.01 \AA^{-1}$ and $k=1.15 \AA^{-1}$ all show a collective mode and the familiar particle-hole continuum. Note that the boundaries of the singleparticle-hole continuum are identical in RPA and CBF. (This is to be contrasted with a theory invoking a $m^{*}$, as the latter changes $\chi^{0}$ and thus the particle-hole continuum). It is clearly seen from the figure that the collective mode in CBF theory is shifted appreciably to lower energies and is about to merge into the continuum at $k=1.15 \AA^{-1}$. Evidently, pair fluctuations are quite able to move the location of the zero sound mode without effecting the particle-hole continuum. Therefore, an adjustment of the effective mass is unnecessary to lower the collective mode, this was also the case ${ }^{18}$ for $3 \mathrm{~d}^{3} \mathrm{He}$.

While the RPA still yields a well separated collective mode at somewhat higher wave numbers, the $\mathrm{CBF}$ phonon has merged into the continuum and $S(k, \omega)$ displays a form similar to the RPA curve in the last part of Fig. 3. The case of the wave number $k=1.34 \AA^{-1}$ is most interesting: In both RPA and CBF, the collective mode has merged with the single-particle-hole continuum. But whereas the RPA spectrum still has a strong peak close to the upper boundary of the continuum, the overall form of the CBF corrected spectrum is now very close to the experimentally observed one.

From these results we conclude that that the essential mechanism determining the shape of the dynamic structure function in ${ }^{3} \mathrm{He}$ is that of fluctuating pair correlations. Our study must, of course, be considered preliminary. A number of physical mechanisms are still unaccounted for:

- We have found that the collective mode can be lowered without introducing an ad-hoc effective mass. Nevertheless a consistent view of multi-pair and single (quasi-)particle properties appears important. In previous work ${ }^{9,10}$ we have taken working formulas from perturbation theory and have combined these with effective interactions generated from CBF theory to compute $m^{*}$. A rigorous justification for this procedure is still lacking.

- In ${ }^{4} \mathrm{He}$, the same approximations used here lead to a significant lowering 

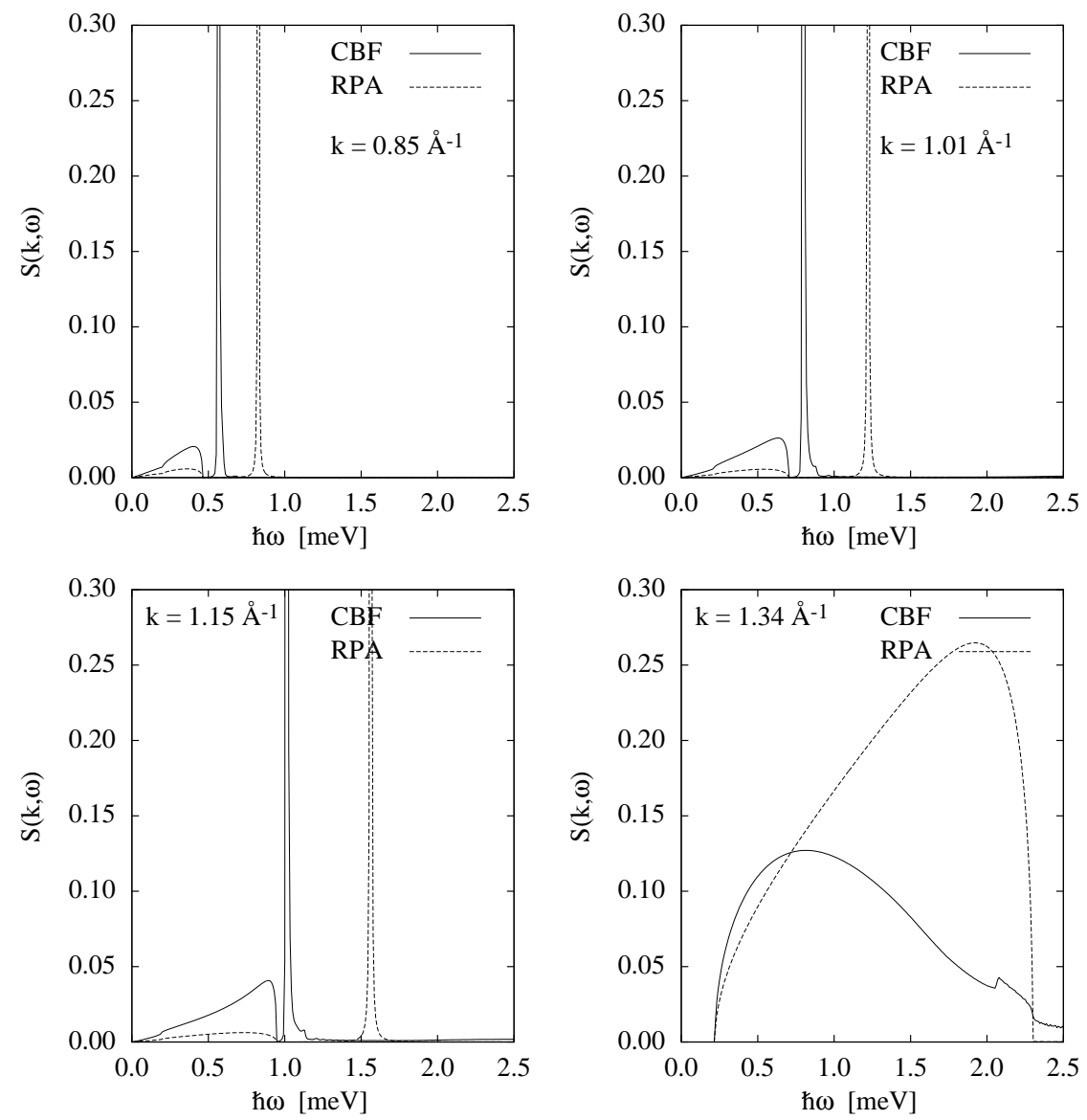

Fig. 3. Dynamic structure factor of a ${ }^{3} \mathrm{He}$ layer at an areal density $n=0.049 \AA^{-2}$ for four different wave numbers as shown in the plots. Full lines: CBF-theory results Dashed lines: RPA results as defined by Eq. (2).

of the phonon-roton spectrum, but still do not reproduce the experiments. A theory with fewer approximations is needed to lower the energy of the collective mode even further. ${ }^{3}$ The same is expected here, note that our collective mode at the experimental wave number is still too high.

- At the experimental wave number $1.1 \AA^{-1} \leq k \leq 1.2 \AA^{-1}$, the particle-hole continuum terminates at about $1 \mathrm{meV}$, whereas the data still show significant strength up to about twice that energy. To explain this feature with single-particle excitations would require an effective mass that is significantly less than 1 , which does not seem realistic. ${ }^{19-21}$ Therefore, a part of the continuum seen in Fig. 1 must be due to multi-pair-excitations. These have been treated in this work as "collective modes" (note that the energy denominator in Eq. (20) contains only collective excitations); clearly this 
approximation must sooner or later be eliminated.

Our first priority is to investigate the effect of pair-fluctuations on quasi-particle excitations in the system within this same formalism, and to combine this aspect of the theory with the multi-pair-theory of the dynamic structure function. In previous work, ${ }^{9,10}$ we have used the so-called G0W approximation without rigorous justification. Such a justification be achieved by studying the time-dependent wave function corresponding to a definite single particle-hole amplitude, i.e. by omitting the sum over $p h$ (only) in the $c_{p h}$ - contribution in Eq. 4. Then one can proceed in exactly the same way as above, leading to a consistent way of studying collective excitations and quasi-particle properties. Work in this direction is in progress.

In summary, the introduction of dynamic multi-pair correlations, so successful in ${ }^{4} \mathrm{He}$, also proves necessary for obtaining the correct phonon dispersion in ${ }^{3} \mathrm{He}$. A promising extension is the inclusion of spin fluctuations, which holds the potential of understanding the large effective mass in ${ }^{3} \mathrm{He}$. As a matter of course the theory can also be applied to other strongly interacting Fermi fluids and to electrons.

\section{Appendix}

We include henceforth the factors $z_{X_{n^{\prime}}^{\prime}}$ in the definition of the correlation amplitudes $u_{(n)}$ and conveniently split off the diagonal parts contained in the correlated EOMs

$$
\begin{aligned}
& N_{X_{n}, X_{n^{\prime}}^{\prime}}=\delta_{n, n^{\prime}} \mathcal{A} \delta_{X_{n}, X_{n^{\prime}}^{\prime}}+\widetilde{N}_{X_{n}, X_{n^{\prime}}^{\prime}} \\
& H_{X_{n}, X_{n^{\prime}}^{\prime}}^{\prime}=\delta_{n, n^{\prime}} e_{X_{n}} \mathcal{A} \delta_{X_{n}, X_{n^{\prime}}^{\prime}}+\widetilde{H}_{X_{n}, X_{n^{\prime}}^{\prime}}^{\prime} .
\end{aligned}
$$

The $n$-particle excitation energies in this definition are essentially the differences of single-particle and -hole energies

$$
e_{X_{n}}=H_{X_{n}, X_{n}}-H_{00}=\sum_{i}\left(e_{p_{i}}-e_{h_{i}}\right)+\mathcal{O}\left(\frac{1}{N}\right)
$$

( $N$ is the particle Number). Defining the correlation amplitude vector as

$$
\mathbf{u} \equiv\left(u_{(1)}, \ldots, u_{(n)}, u_{(1)}^{*}, \ldots, u_{(n)}^{*}\right) \equiv\left(c_{p^{\prime \prime} h^{\prime \prime}}, d_{p^{\prime \prime} p^{\prime \prime \prime}, h^{\prime \prime} h^{\prime \prime \prime}}, c_{p^{\prime \prime} h^{\prime \prime}}^{*}, d_{p^{\prime \prime} p^{\prime \prime \prime}, h^{\prime \prime} h^{\prime \prime \prime}}^{*}\right)
$$

the correlated EOMs can be cast into matrix form

$$
\left[-\left(\frac{\underline{\underline{\mathbf{M}}}}{\underline{\underline{\mathbf{O}}}} \underline{\underline{\underline{\mathbf{M}}}}\right) \frac{\hbar}{i} \frac{\partial}{\partial t}-\left(\begin{array}{l}
\underline{\underline{\Omega_{0}}} \underline{\underline{\underline{\mathbf{O}}}} \underline{\underline{\underline{\Omega_{0}}}}
\end{array}\right)\right] \cdot \mathbf{u}=\left(\underline{\underline{\underline{\mathbf{A}}}} \underline{\underline{\underline{\mathbf{B}}}} \underline{\underline{\underline{\mathbf{A}^{*}}}}\right) \cdot \mathbf{u},
$$

where $\underline{\underline{\mathrm{O}}}$ is the $2 \times 2$ zero matrix, $\underline{\underline{\Omega_{0}}}$ contains the excitation energies

$$
\underline{\underline{\underline{\Omega_{0}}}}:=\left(\begin{array}{cc}
\left(e_{p}-e_{h}\right) \delta_{p p^{\prime \prime}} & 0 \\
0 & \left(e_{p}-e_{h}+e_{p^{\prime}}-e_{h^{\prime}}\right) \mathcal{A} \delta_{\substack{p p^{\prime \prime} \\
h h^{\prime \prime}}} \delta_{\substack{p^{\prime} p^{\prime \prime \prime} \\
h^{\prime} h^{\prime \prime \prime}}}
\end{array}\right)
$$

with

$$
\mathcal{A} \delta_{\substack{p p^{\prime \prime} \\ h h^{\prime \prime}}} \delta_{\substack{p^{\prime} p^{\prime \prime \prime} \\ h^{\prime} h^{\prime \prime \prime}}}:=\delta_{\substack{p p^{\prime \prime} \\ h h^{\prime \prime}}} \delta_{p^{\prime} p^{\prime \prime \prime \prime} h^{\prime \prime \prime}}-\delta_{\substack{p p^{\prime \prime \prime} \\ h h^{\prime \prime}}} \delta_{\substack{p^{\prime} p^{\prime \prime} \\ h^{\prime} h^{\prime \prime \prime}}}-\delta_{\substack{p p^{\prime \prime} \\ h h^{\prime \prime \prime}}} \sum_{\substack{p^{\prime} p^{\prime \prime \prime \prime} h^{\prime \prime} \\ h^{\prime}}}+\delta_{\substack{p p^{\prime \prime \prime} \\ h h^{\prime \prime \prime}}} \delta_{\substack{p^{\prime} p^{\prime \prime} \\ h^{\prime} h^{\prime \prime}}} .
$$


The time derivative terms have factors arising from the normalization overlap integrals contained in $\underline{\underline{\mathbf{M}}}$

$$
\begin{aligned}
& \underline{\underline{\mathbf{M}}}=\left(\begin{array}{cc}
\delta_{p p^{\prime \prime}} & 0 \\
h h^{\prime \prime} & \mathcal{A} \delta_{\substack{p p^{\prime \prime} \\
h h^{\prime \prime}}} \delta_{\substack{p^{\prime} p^{\prime \prime \prime} \\
h^{\prime} h^{\prime \prime \prime}}}
\end{array}\right)+ \\
& \left(\begin{array}{cc}
\widetilde{N}_{p h, p^{\prime \prime} h^{\prime \prime}} & \frac{1}{2} N_{p h, p^{\prime \prime} h^{\prime \prime} p^{\prime \prime \prime} h^{\prime \prime \prime}} \\
N_{p h p^{\prime} h^{\prime}, p^{\prime \prime} h^{\prime \prime}} & \frac{1}{2}\left[\widetilde{N}_{p h p^{\prime} h^{\prime}, p^{\prime \prime} h^{\prime \prime} p^{\prime \prime \prime} h^{\prime \prime \prime}}-N_{0, p h p^{\prime} h^{\prime}} N_{0, p^{\prime \prime} h^{\prime \prime} p^{\prime \prime \prime} h^{\prime \prime \prime}}\right]
\end{array}\right),
\end{aligned}
$$

(in the bulk system $N_{p h, 0}=0$ ). Finally, the dynamics is governed by the Hamiltonian contributions

$$
\begin{aligned}
& \underline{\underline{\underline{\mathbf{A}}}}:=\left(\begin{array}{cc}
\widetilde{H}_{p h, p^{\prime \prime} h^{\prime \prime}}^{\prime} & \frac{1}{2} H_{p h, p^{\prime \prime} h^{\prime \prime} p^{\prime \prime \prime} h^{\prime \prime \prime}} \\
H_{p h p^{\prime} h^{\prime}, p^{\prime \prime} h^{\prime \prime}}^{\prime} & \frac{1}{2} \widetilde{H}_{p h p^{\prime} h^{\prime}, p^{\prime \prime} h^{\prime \prime} p^{\prime \prime \prime} h^{\prime \prime \prime}}^{\prime}
\end{array}\right) \\
& \underline{\underline{\mathbf{B}}}:=\left(\begin{array}{cc}
H_{p h p^{\prime \prime} h^{\prime \prime}, 0}^{\prime} & \frac{1}{2} H_{p h p^{\prime \prime} h^{\prime \prime} p^{\prime \prime \prime} h^{\prime \prime \prime}, 0} \\
\bar{H}_{p h p^{\prime} h^{\prime} p^{\prime \prime} h^{\prime \prime}, 0}^{\prime} & \frac{1}{2} \bar{H}_{p h p^{\prime} h^{\prime} p^{\prime \prime} h^{\prime \prime} p^{\prime \prime \prime} h^{\prime \prime \prime}, 0}^{\prime}
\end{array}\right) \\
& \bar{H}_{p h p^{\prime} h^{\prime} p^{\prime \prime} h^{\prime \prime}, 0}^{\prime} \equiv\left[H_{p h p^{\prime} h^{\prime} p^{\prime \prime} h^{\prime \prime}, 0}^{\prime}-N_{p h p^{\prime} h^{\prime}, 0} H_{p^{\prime \prime} h^{\prime \prime}, 0}^{\prime}\right] \\
& \bar{H}_{p h p^{\prime} h^{\prime} p^{\prime \prime} h^{\prime \prime} p^{\prime \prime \prime} h^{\prime \prime \prime}, 0}^{\prime} \equiv\left[H_{p h p^{\prime} h^{\prime} p^{\prime \prime} h^{\prime \prime} p^{\prime \prime \prime} h^{\prime \prime \prime}, 0}^{\prime}-N_{p h p^{\prime} h^{\prime}, 0} H_{p^{\prime \prime} h^{\prime \prime} p^{\prime \prime \prime} h^{\prime \prime \prime}, 0}^{\prime}\right]
\end{aligned}
$$

Obviously, Eq. (27) is similar to the corresponding dynamic CBF equations containing single-particle excitations only ${ }^{12}$, the matrices $\underline{\underline{\mathbf{A}}}$ and in particular $\underline{\underline{\mathbf{B}}}$, however, being much more intricate.

\section{Acknowledgments}

This work was supported by the Austrian Science Fund (FWF) project P18134N08. We thank V. Grau, J. Boronat and J. Casulleras for providing the Monte Carlo data ${ }^{17}$ of structure functions in two-dimensional ${ }^{3} \mathrm{He}$.

\section{References}

1. E. Feenberg, Theory of Quantum Fluids (Academic, New York, 1969).

2. B. E. Clements, E. Krotscheck and C. J. Tymczak, Phys. Rev. B 53, 12253 (1996).

3. V. Apaja and M. Saarela, Phys. Rev. B 57, p. 5358 (1998).

4. V. Apaja and E. Krotscheck, A microscopic view of confined quantum liquids, in $M i$ croscopic Approaches to Quantum Liquids in Confined Geometries, eds. E. Krotscheck and J. Navarro (World Scientific, Singapore, 2002) pp. 205-268.

5. R. P. Feynman and M. Cohen, Phys. Rev. 102, 1189 (1956).

6. N. Iwamoto and D. Pines, Phys. Rev. B 29, 3924 (1984).

7. K. S. Singwi and M. P. Tosi, Solid State Phys. 36, 177 (1981).

8. H. R. Glyde, B. Fåk, N. H. van Dijk, H. Godfrin, K. Guckelsberger and R. Scherm, Phys. Rev. B 61, 1421 (2000).

9. B. L. Friman and E. Krotscheck, Phys. Rev. Lett. 49, 1705 (1982). 
10. E. Krotscheck and J. Springer, J. Low Temp. Phys. 132, p. 281 (2003).

11. H. Godfrin, M. Meschke and H. J. Lauter, private communication (2003).

12. E. Krotscheck, Theory of correlated basis functions, in Introduction to Modern Methods of Quantum Many-Body Theory and their Applications, eds. A. Fabrocini, S. Fantoni and E. Krotscheck, Advances in Quantum Many-Body Theory, Vol. 7 (World Scientific, Singapore, 2002) pp. 267-330.

13. J. M. C. Chen, J. W. Clark and D. G. Sandler, Z. Physik A 305, 223 (1982).

14. E. Krotscheck, Phys. Rev. A 26, 3536 (1982).

15. E. Krotscheck and J. W. Clark, Nucl. Phys. A 328, 73 (1979).

16. K. E. Schörkhuber, Multi-particle-hole excitations in many-body fermi systems, PhD thesis, Institut für Theoretische Physik, Johannes Kepler University, Linz, Austria2002.

17. V. Grau, J. Boronat and J. Casulleras, Phys. Rev. Lett. 89, 045301 (2002).

18. H. M. Böhm, E. Krotscheck, K. Schörkhuber and J. Springer, Pair excitations in fermi fluids, in Proceedings of the XXIV th International Conference on Low Temperature Physics, ed. Y. Takano (AIP, New York, 2006).

19. D. S. Greywall, Phys. Rev. B 41, 1842 (1990).

20. K.-D. Morhard, C. Bäuerle, J. Bossy, Y. Bunkov, S. N. Fisher and H. Godfrin, Phys. Rev. B 53, 2658 (1996).

21. A. Casey, H. Patel, J. Nyéki, B. P. Cowan and J. Saunders, Phys. Rev. Lett. 90, 115301 (2003). 